\title{
Qualidade do serviço of talmológico prestado aos pacientes ambulatoriais do Sistema Único de Saúde - SUS
}

\author{
Ophthalmological service quality offered tooutpatients of the Public Healthcare System
}

\author{
Benigno Vicente Santos Hercos ${ }^{1}$ \\ Adriana Berezovsky ${ }^{2}$
}

${ }^{1}$ Doutor em Medicina, subárea oftalmologia, Universidade Autônoma de Barcelona - Espanha. Departamento de Oftalmologia - Universidade Federal de São Paulo UNIFESP - São Paulo (SP) - Brasil.

2 Doutora em Ciências e Professora Adjunto do Departamento de Oftalmologia pela UNIFESP - São Paulo (SP) Brasil.

Endereço para correspondência: Rua Antares, 145/ 202 - Belo Horizonte (MG) CEP 30360 -110

E-mail: b-hercos@uol.com.br

Recebido para publicação em 27.10.2004

Versão revisada recebida em 22.11.2005

Aprovação em 02.12.2005

Nota Editorial: Depois de concluída a análise do artigo sob sigilo editorial e com a anuência da Dra. Silvia Prado Smit Kitadai sobre a divulgação de seu nome como revisora, agradecemos sua participação neste processo.

\begin{tabular}{|l|}
\hline RESUMO \\
\hline Objetivos: Identificar a percepção da qualidade dos serviços oftalmológicos \\
prestados aos pacientes ambulatoriais do Sistema Único de Saúde - SUS \\
- e detectar quais ações são percebidas como necessárias e prioritárias para \\
melhorar a sua qualidade. Métodos: Foi realizado estudo descritivo quan- \\
titativo de 100 pacientes ambulatoriais do SUS, submetidos a exame \\
oftalmológico na Fundação Hilton Rocha, em Belo Horizonte - MG, no \\
período de 1 de junho a 30 de julho de 2004. Realizaram-se entrevistas \\
pessoais, mediante a aplicação de dois questionários estruturados adap- \\
tados da escala SERVQUAL modificada. Essa escala foi adaptada à \\
realidade da instituição estudada. Resultados: A escala SERVQUAL \\
adaptada foi submetida à validação estatística apresentando adequado \\
índice de consistência interna. Em termos gerais, detectou-se ligeira \\
insatisfação geral com a qualidade do atendimento oftalmológico. Os \\
entrevistados deram maior importância à segurança e à confiabilidade. \\
Detectou-se o maior grau de insatisfaçãona confiabilidade, principalmente \\
em relação ao cumprimento das atividades nos horários marcados e em \\
relação à execução dos serviços no prazo prometido. Conclusões: A \\
instituição deve planejar e executar ações que levem a melhora geral da \\
satisfação de seus pacientes com a qualidade do serviço recebido, prin- \\
cipalmente no aspecto confiabilidade. A monitorização da qualidade do \\
serviço pelo emprego periódico da escala SERVQUAL permitiria não só \\
planejar estratégias precisas de intervenção de alta efetividade neste e em \\
outros serviços de saúde, como também permitiria monitorizar a resposta \\
a essas ações, contribuindo, dessa forma, para a melhora da qualidade do \\
serviço no sistema como um todo.
\end{tabular}

Descritores: Marketing de serviços de saúde; Oftalmologia; Qualidade dos cuidados de saúde; Indicadores de qualidade em assistência à saúde; Assistência ambulatorial; Questionários/utilização

\section{INTRODUÇÃOO}

Marketing é o conjunto de atividades humanas destinado a atender os desejos e as necessidades dos consumidores por meio de processos de troca, utilizando "ferramentas" específicas, como a propaganda, a promoção de vendas, a pesquisa de marketing, entre outras ${ }^{(1)}$.

Para planejar e administrar em marketing, é necessário ter informações seguras sobre a situação de $\operatorname{mercado}^{(1)}$.

Nos últimos anos, os estudos sobre a satisfação do consumidor absorveram parte considerável dos esforços dos pesquisadores em marketing, dos institutos de pesquisa, dos órgãos governamentais e das empresas interessadas em implementar programas de qualidade total ${ }^{(2)}$. 
O setor de serviços em que se insere o setor de saúde possui algumas particularidades em relação à industria e ao comércio. A prestação de serviços inclui expectativas dos clientes, muito mais subjetivas e variáveis que a manufatura de produtos. Lida com sentimentos como empatia e sensibilidade $^{(3)}$. Lida com os aspectos da qualidade mais difíceis de serem alcançados - os aspectos intangíveis ${ }^{(3-4)}$.

As informações sobre a qualidade de um serviço necessitam de prévia pesquisa. Alguns autores recomendam a utilização de um catálogo de técnicas de pesquisa conhecido como sistema de informações sobre qualidade de serviço ${ }^{(5)}$. Esse sistema apresenta várias abordagens, e, dentre estas, interessa-nos, neste estudo, a pesquisa total de mercado utilizando a escala SERVQUAL, que é uma escala de avaliação de qualidade que vem sendo bastante utilizada ${ }^{(6)}$ e que permite avaliar as cinco dimensões da qualidade de serviço: tangibilidade, confiabilidade, atendimento, segurança e empatia ${ }^{(6-7)}$. A escala SERVQUAL é uma técnica de pesquisa de marketing mundialmente utilizada para avaliar a qualidade do serviço através da determinação das expectativas e percepções dos pacientes ${ }^{(8)}$. A aplicação da escala SERVQUAL para avaliar a qualidade de serviços de saúde já está bem documentada e é um instrumento autorizado para esse $\mathrm{fim}^{(9-10)}$. Na área temática de Marketing, avaliando-se a qualidade de serviço e utilizando-se a escala SERVQUAL, é importante citar o trabalho de Brasil ${ }^{(11)}$.

Por fim, em que pese a crescente importância da noção de qualidade de serviço, pouca pesquisa acadêmica tem focalizado as condições de sua mensuração, algo indispensável para fazer avançar a compreensão e aplicabilidade desse domínio. Os objetivos de Brasil eram verificar a dimensionalidade e a operacionalização do instrumento SERVQUAL no contexto dos serviços hospitalares.

O ponto de partida deste trabalho é a própria escala SERVQUAL, desenvolvida ao longo de vários anos de pesquisas empíricas nos Estados Unidos por Parasuraman, et $\mathrm{al}^{(12-13)}$.

Este trabalho tem como objetivos identificar a percepção da qualidade do serviço oftalmológico prestado aos pacientes ambulatoriais do SUS pela Fundação Hilton Rocha e, a partir dessas informações, detectar quais ações são percebidas como necessárias e prioritárias para melhorar a qualidade do atendimento.

\section{MÉTODOS}

Foi realizado um estudo descritivo quantitativo de 100 pacientes ambulatoriais do SUS submetidos a exame oftalmológico na Fundação Hilton Rocha - Belo Horizonte - MG, no período de 1 de junho a 30 de julho de 2004. Foram incluídos pacientes de ambos os sexos e com idades entre 18 e 85 anos. Realizaram-se entrevistas pessoais, mediante a aplicação de dois questionários estruturados adaptados da escala SERVQUAL modificada (Figura 1), com 23 perguntas (itens) cada um, o que permitiu avaliar a qualidade do serviço através da medida das expectativas e percepções sobre dimensões críticas da qualidade. A distribuição e a aplicação dos questionários aos pacientes foram feitas por uma entrevistadora treinada para esse serviço.
Pediu-se, antes da consulta, que os pacientes preenchessem uma série de escalas que mediram suas expectativas em relação ao atendimento oftalmológico que gostariam de receber, sobre um amplo leque de características específicas do serviço, avaliando as cinco dimensões da qualidade (Tangibilidade - itens 1- 4, confiabilidade - itens 5- 9, atendimento itens 10-13, segurança - itens 14-17 e empatia - itens 18-22). Dentro de cada uma das cinco dimensões, encontram-se vários itens medidos em uma escala de avaliação de 7 pontos, que vão desde concordo inteiramente $=7$ pontos a discordo inteiramente $=1$ ponto. Só os pontos extremos de cada escala foram rotulados. Aplicou-se conjuntamente um pequeno questionário (alocação de pontos) para determinar qual a importância de cada uma das cinco dimensões da qualidade de serviço para os entrevistados. Após a consulta, pediu-se aos pacientes que registrassem suas percepções sobre o atendimento recebido preenchendo o mesmo questionário.

As notas do desempenho percebido inferiores às das expectativas significaram um resultado negativo, o que é sinal de que a qualidade percebida está abaixo do esperado; ao contrário, indicam que a qualidade percebida está acima do esperado; e a nota próxima de zero é sinal de que a qualidade percebida é satisfatória.

Para o cálculo do Escore de Satisfação do Consumidor utilizou-se a fórmula ${ }^{(2)}$ :

$$
E S C_{j i}=\frac{\sum_{i=1}^{n_{j}} P_{i}-E_{i} E_{i}}{n_{j}}-
$$

onde, $P_{\mathrm{i}}$ é a percepção avaliada na questão $i$

$E_{\mathrm{i}}$ é a situação ideal avaliada na questão $i$

$n_{\mathrm{j}}$ é o número de questões pertencentes ao indicador $j$

Além dos dados obtidos dos questionários, foram também analisados: sexo, idade e escolaridade dos pacientes. Analisaram-se aspectos da mensuração da qualidade de serviço relativos à fidedignidade, validade de convergência e à dimensionalidade dessa versão modificada da escala SERVQUAL.

As informações recebidas foram tabuladas e analisadas, utilizando-se o teste de Kruskal-Wallis para analisar a influência dos fatores de interesse na avaliação da qualidade do serviço, e utilizando-se também a análise de conglomerados (análise cluster) para determinar os perfis diferenciados dos entrevistados quanto ao grau de satisfação com o serviço. Foram considerados significativos os valores de $\mathrm{p}<5$, havendo, portanto, pelo menos $95 \%$ de confiança nas conclusões apresentadas. Todas as análises estatísticas foram realizadas utilizando-se o software SAS versão 5 (SAS INSTITUT INC.), que forneceu a análise descritiva dos dados.

\section{RESULTADOS}

Apresentam-se os resultados dos testes utilizados para a validação estatística da escala utilizada, a seguir. O Alfa de Cronbach relativo a toda a escala foi de 0,93 , sendo superior a 


\begin{tabular}{|c|c|c|c|c|c|c|c|c|c|c|c|c|c|c|}
\hline \multirow[t]{2}{*}{ Característica } & \multicolumn{7}{|c|}{ Atendimento ideal } & \multicolumn{7}{|c|}{ Atendimento recebido } \\
\hline & 1 & 2 & 3 & 4 & 5 & 6 & 7 & 1 & 2 & 3 & 4 & 5 & 6 & 7 \\
\hline 1. A Fundação deve ter equipamentos conservados e modernos. & & & & & & & & & & & & & & \\
\hline $\begin{array}{l}\text { 2. Instalações físicas da Fundação devem ser visualmente bonitas } \\
\text { e agradáveis. }\end{array}$ & & & & & & & & & & & & & & \\
\hline $\begin{array}{l}\text { 3. A equipe (médicos, enfermeiros e funcionários administrativos) da } \\
\text { Fundação devem ter aparência e trajes bem cuidados, de acordo } \\
\text { com as características do ambiente de trabalho. }\end{array}$ & & & & & & & & & & & & & & \\
\hline $\begin{array}{l}\text { 4. Relatórios e demais documentos entregues ao paciente na } \\
\text { Fundação devem ser de fácil compreensão e visualmente atrativos. }\end{array}$ & & & & & & & & & & & & & & \\
\hline 5. A Fundação deve realizar suas atividades no horário marcado. & & & & & & & & & & & & & & \\
\hline $\begin{array}{l}\text { 6. A Fundação deve demonstrar interesse sincero em resolver os } \\
\text { problemas do paciente. }\end{array}$ & & & & & & & & & & & & & & \\
\hline $\begin{array}{l}\text { 7. A Fundação deve executar os serviços e procedimentos de forma } \\
\text { correta da primeira vez, não ocasionando retrabalho. }\end{array}$ & & & & & & & & & & & & & & \\
\hline $\begin{array}{l}\text { 8. A Fundação deve prestar seus serviços no prazo de } \\
\text { execução prometido. }\end{array}$ & & & & & & & & & & & & & & \\
\hline $\begin{array}{l}\text { 9. A Fundação deve apresentar relatórios, documentos e informações } \\
\text { sobre o paciente sem erros. }\end{array}$ & & & & & & & & & & & & & & \\
\hline $\begin{array}{l}\text { 10. Na Fundação a equipe (médicos, enfermeiros e funcionários } \\
\text { administrativos) deve informar aos pacientes com exatidão quando } \\
\text { os serviços serão executados. }\end{array}$ & & & & & & & & & & & & & & \\
\hline $\begin{array}{l}\text { 11. Na Fundação a equipe (médicos, enfermeiros e funcionários } \\
\text { administrativos) deve atender prontamente seus pacientes. }\end{array}$ & & & & & & & & & & & & & & \\
\hline $\begin{array}{l}\text { 12. Na Fundação a equipe (médicos, enfermeiros e funcionários } \\
\text { administrativos) deve procurar ajudar seus pacientes. }\end{array}$ & & & & & & & & & & & & & & \\
\hline $\begin{array}{l}\text { 13. Na Fundação a equipe (médicos, enfermeiros e funcionários } \\
\text { administrativos) deve estar disponível para atender aos } \\
\text { pedidos do paciente. }\end{array}$ & & & & & & & & & & & & & & \\
\hline $\begin{array}{l}\text { 14. Na Fundação o comportamento da equipe (médicos, enfermeiros } \\
\text { e funcionários administrativos) deve transmitir confiança } \\
\text { aos pacientes. }\end{array}$ & & & & & & & & & & & & & & \\
\hline $\begin{array}{l}\text { 15. Pacientes da Fundação devem sentir-se seguros em utilizar } \\
\text { os seus serviços. }\end{array}$ & & & & & & & & & & & & & & \\
\hline $\begin{array}{l}\text { 16. Na Fundação a equipe (médicos, enfermeiros e funcionários } \\
\text { administrativos) deve ser educada e cortês com os seus pacientes. }\end{array}$ & & & & & & & & & & & & & & \\
\hline $\begin{array}{l}\text { 17. Na Fundação a equipe (médicos, enfermeiros e funcionários } \\
\text { administrativos) deve ter conhecimento adequado para responder } \\
\text { as perguntas dos pacientes. }\end{array}$ & & & & & & & & & & & & & & \\
\hline 18. Na Fundação a equipe deve dar atenção individualizada ao cliente. & & & & & & & & & & & & & & \\
\hline $\begin{array}{l}\text { 19. A Fundação deve funcionar em horários adequados aos } \\
\text { seus pacientes. }\end{array}$ & & & & & & & & & & & & & & \\
\hline $\begin{array}{l}\text { 20. A Fundação deve ter uma equipe que dê atenção pessoal aos } \\
\text { seus pacientes. }\end{array}$ & & & & & & & & & & & & & & \\
\hline 21. A Fundação deve priorizar os interesses do paciente. & & & & & & & & & & & & & & \\
\hline $\begin{array}{l}\text { 22. Na Fundação a equipe deve entender as necessidades } \\
\text { específicas de seus pacientes. }\end{array}$ & & & & & & & & & & & & & & \\
\hline $\begin{array}{l}\text { 23. Na Fundação a qualidade geral do atendimento oftalmológico deve } \\
\text { ser satisfatória }\end{array}$ & & & & & & & & & & & & & & \\
\hline
\end{tabular}

\section{Alocação de pontos}

Instruções: Cinco características do atendimento oftalmológico ambulatorial na Fundação estão listadas abaixo.

Indique a importância de cada uma destas características na avaliação da qualidade do atendimento oftalmológico na fundação. Distribua um total de 100 pontos entre as cinco características, de acordo com o grau de importância em sua avaliação - quanto mais importante for a característica, mais pontos devem ser usados na avaliação.

Assegure que o total da avaliação das cinco características some 100 pontos.

\section{Características}

1. A aparência das instalações físicas, pessoal, materiais de comunicação e equipamentos da Fundação.

Pontos

pontos

2. A execução dos serviços de forma precisa, confiável e segura pela Fundação.

3. A ajuda aos pacientes e o pronto atendimento às suas necessidades pela Fundação.

4. O conhecimento, a cortesia e a confiabilidade transmitida pela equipe (médicos, enfermeiras e funcionários administrativos) aos pacientes da Fundação.

5. A atenção individualizada aos pacientes pela equipe da Fundação.

Total pontos

pontos

pontos

100 pontos

Dados do Entrevistado: Escolaridade: ( ) superior; ( ) médio - $5^{\underline{a}}$ do $1^{\circ}$ grau ao $3^{\circ}$ ano do $2^{\circ}$ grau; ( ) primário - $1^{\underline{a}}$ a $4^{\underline{a}}$ séries do $1^{\circ}$ grau; ( ) analfabeto Idade: Sexo:

Figura 1 - Modelo do questionário da pequisa (Fonte: adaptado da escala SERVQUAL modificada) 
0,60 em todas as dimensões avaliadas, o que indica uma consistência interna satisfatória para a escala utilizada verificando a sua fidedignidade ${ }^{(14-16)}$. A análise da relação entre a avaliação geral e as escalas foram significativas com $\mathrm{p}<0,001$ para todas as dimensões da escala, o que demonstra assim a validade convergente da escala utilizada. A dimensionalidade da escala foi avaliada utilizando-se a análise fatorial, porém não foi verificada. Demonstrou-se existência de relações entre todas as escalas avaliadas, por exemplo, entre tangibilidade e confiabilidade. Observa-se também uma correlação positiva, ou seja, o aumento da satisfação em uma escala vem acompanhado do aumento da satisfação na outra escala.

Do total dos entrevistados, $66 \%$ pertenciam ao sexo feminino e os demais $34 \%$ ao sexo masculino. A idade da amostra variou de 18 a 78 anos, com uma média igual a 36 anos e o desvio padrão igual a 15 anos. Quanto ao nível de escolaridade, houve um predomínio do nível médio (67\%), seguido pelo primário (25\%), 6\% tinham nível superior e $2 \%$ eram analfabetos.

A tabela 1 mostra que os entrevistados estão mais insatisfeitos quanto à confiabilidade $(-1,7)$, uma vez que esse indicador apresentou uma distância maior na média entre a avaliação da instituição em questão e o que seria uma instituição ideal. Os demais indicadores demonstraram resultados inferiores e similares, demonstrando uma menor insatisfação. A avaliação geral do serviço prestado pela instituição variou de $-5,8$ a 1,4 com média igual a -1,1 demonstrando uma discreta insatisfação geral.

A tabela 2 mostra que os entrevistados dão maior importância para a confiabilidade $(22,8)$ e a segurança $(24,2)$ e menor importância para os indicadores que avaliam a tangibilidade, atendimento e empatia.

\begin{tabular}{|c|c|c|c|c|c|}
\hline \multirow[b]{2}{*}{ Indicadores } & \multicolumn{5}{|c|}{ Medidas descritivas } \\
\hline & Mínimo & Máximo & Mediana & Média & D.p. \\
\hline Tangibilidade & $-5,8$ & 2,8 & $-0,8$ & $-1,0$ & 1,4 \\
\hline Confiabilidade & $-6,0$ & 1,8 & $-1,4$ & $-1,7$ & 1,5 \\
\hline Atendimento & $-5,8$ & 2,8 & $-0,8$ & $-1,1$ & 1,7 \\
\hline Segurança & $-5,8$ & 2,0 & $-0,4$ & $-0,8$ & 1,4 \\
\hline Empatia & $-6,0$ & 1,8 & $-0,4$ & $-0,8$ & 1,5 \\
\hline Avaliação geral & $-5,8$ & 1,4 & $-0,8$ & $-1,1$ & 1,3 \\
\hline
\end{tabular}

\begin{tabular}{|c|c|c|c|c|c|}
\hline \multirow[b]{2}{*}{ Indicadores } & \multicolumn{5}{|c|}{ Medidas descritivas } \\
\hline & Mínimo & Máximo & Mediana & Média & D.p. \\
\hline Tangibilidade & 0,0 & 70,0 & 20,0 & 19,2 & 11,7 \\
\hline Confiabilidade & 0,0 & 60,0 & 20,0 & 22,8 & 11,1 \\
\hline Atendimento & 0,0 & 50,0 & 20,0 & 17,1 & 8,9 \\
\hline Segurança & 0,0 & 60,0 & 20,0 & 24,2 & 10,7 \\
\hline Empatia & 0,0 & 50,0 & 20,0 & 16,8 & 8,5 \\
\hline \multicolumn{6}{|c|}{ Nota: $p<0,001 \rightarrow$ Teste de Friedman } \\
\hline
\end{tabular}

Ao avaliar-se especificamente cada um dos itens dos indicadores da qualidade de serviço: tangibilidade, atendimento, segurança e empatia, observou-se que a diferença entre a avaliação da instituição e a situação ideal para cada um dos itens foi semelhante, situando-se em valor médio de - 1,0 , o que demonstra discreta insatisfação em relação a estes indicadores. Já com relação ao indicador confiabilidade, conforme se pode observar na tabela 3 , é nítida uma maior insatisfação em relação ao cumprimento das atividades nos horários marcados (item 5), pois o valor da diferença é de -3,3. Em relação à execução dos serviços no prazo prometido (item 8 ), o valor da diferença é de $-2,1$.

$\mathrm{O}$ indicador segurança merece ser destacado por ter sido considerado o mais importante pela maioria dos entrevistados, conforme se pode observar na tabela 4 , e, quanto à segurança, o grau de insatisfação dos usuários foi muito baixo, sendo menor ainda com relação ao item 17, cuja diferença foi de -0,7: a equipe deve ter conhecimento adequado para responder às perguntas dos pacientes.

A análise dos resultados mostra que o sexo e a idade não exercem influências significativas na avaliação dos indicadores da qualidade de serviço. Com relação ao nível de escolaridade, no entanto, os dados encontrados indicam uma tendência em que pacientes com nível de escolaridade superior $(\mathrm{p}=0,07)$ estão mais insatisfeitos com o aspecto do atendimento recebido.

Baseando-se na Análise de Conglomerados de todos os entrevistados avaliados, foi possível identificar 3 grupos distintos: um primeiro grupo com 5 entrevistados, um segundo com 34 entrevistados e um terceiro com 61 entrevistados.

Comparando-se os grupos em relação às cinco dimensões, observaram-se diferenças significativas. Para a tangibilidade, foram verificadas similaridades entre o grupo 1 e o grupo 2, e estes se apresentaram mais insatisfeitos do que o grupo 3 . Para as demais dimensões, observaram-se diferenças significativas entre os três grupos: o grupo 1, o mais insatisfeito; e o grupo 3, o menos insatisfeito. Portanto, o grupo 3, que é o que compreende o maior número de entrevistados, corresponde ao grupo com menor grau de insatisfação.

A caracterização dos grupos de pacientes quanto à escolaridade, ao sexo e à faixa etária mostra que os pacientes do grupo 1 (mais insatisfeitos com a qualidade do serviço) eram, em sua maioria, do sexo masculino (60\%), a faixa etária predominante era de 41 a 50 anos (40\%) e $80 \%$ tinham nível de escolaridade primário/analfabeto ou médio. Já os pacientes do grupo 3 (menos insatisfeitos) eram em sua maioria do sexo feminino $(67,2 \%)$, a faixa etária predominante era de 21 a 30 anos e 72,1\% tinham nível de escolaridade médio.

\section{DISCUSSÃO}

Os dados obtidos neste estudo mostraram que essa escala SERVQUAL adaptada apresentou um adequado índice de consistência interna, tendo sido observada uma distância (gap) entre a percepção da qualidade do serviço prestado e as 


\begin{tabular}{|c|c|c|c|}
\hline \multicolumn{4}{|c|}{ Medida da avaliação da qualidade do serviço } \\
\hline Itens do indicador confiabilidade & $\begin{array}{l}\text { Percebido } \\
\text { (recebido) }\end{array}$ & $\begin{array}{c}\text { Ideal } \\
\text { (expectativa) }\end{array}$ & $\begin{array}{l}\text { Resultado (desempenho } \\
\text { percebido menos ideal) }\end{array}$ \\
\hline 5. A Fundação deve realizar suas atividades no horário marcado. & 2,9 & 6,2 & $-3,3$ \\
\hline $\begin{array}{l}\text { 6. A Fundação deve demonstrar interesse sincero em resolver os } \\
\text { problemas do paciente. }\end{array}$ & 4,6 & 6,0 & $-1,4$ \\
\hline $\begin{array}{l}\text { 7. A Fundação deve executar os serviços e procedimentos de forma } \\
\text { correta da primeira vez, não ocasionando retrabalho. }\end{array}$ & 4,6 & 5,6 & $-1,0$ \\
\hline 8. A Fundação deve prestar seus serviços no prazo de execução prometido. & 3,9 & 6,0 & $-2,1$ \\
\hline $\begin{array}{l}\text { 9. A Fundação deve apresentar relatórios, documentos e informações } \\
\text { sobre o paciente sem erros. }\end{array}$ & 5,0 & 6,0 & $-1,0$ \\
\hline
\end{tabular}

\begin{tabular}{|lcc|}
\hline \multicolumn{1}{|c|}{ Tabela 4. Desempenho dos atributos(itens) do indicador segurança em relação à qualidade do serviço } \\
Medida da avaliação da qualidade do serviço
\end{tabular}

expectativas dos clientes sobre o mesmo nas cinco dimensões estudadas: tangibilidade, confiabilidade, atendimento, segurança e empatia. Dessa forma, conclui-se que essa metodologia é perfeitamente aplicável nas áreas de serviços de saúde, fato este que é compatível com a literatura internacional ${ }^{(9-10)}$.

Em termos gerais, detectou-se uma insatisfação geral muito discreta em relação à qualidade do atendimento oftalmológico recebido pelos pacientes ambulatoriais do SUS, na instituição avaliada, observando-se uma tendência de pacientes com maior nível de escolaridade manifestarem mais freqüentemente sua insatisfação com relação ao atendimento recebido. Alguns autores explicam este discreto grau de insatisfação por, na realidade, existir uma tendência de os usuários de serviços de saúde apresentarem-se satisfeitos ${ }^{(17-23)}$. Alguns estudos mostram que essa tendência inicial de os usuários apresentarem-se satisfeitos desaparece ao se aprofundarem mais as opiniões desses pacientes, principalmente aquelas a respeito da atenção que recebem dos médicos. Essa insatisfação dos usuários manifesta-se, em especial, por não obterem do médico informação suficiente sobre o seu problema ou pelo fato de o médico não lhes dedicar tempo suficiente na consul$\mathrm{ta}^{(10)}$. A qualidade da relação médico-paciente é o critério que se correlaciona com mais força com a satisfação geral dos usuários $^{(10)}$. Outros autores, ao refletirem sobre esse grau de satisfação em pacientes ambulatoriais, aventam a hipótese de que, apesar das condições objetivas conduzirem a maior grau de insatisfação, a percepção do benefício recebido pelo serviço prestado é tão grande em comparação com as carências e limitações do serviço, que o paciente expressa o benefício recebido como satisfação, apesar de existirem manifestações objetivas de desconforto ${ }^{(22)}$. Muitos autores acreditam que o nível de satisfação encontrado nessas pesquisas reflete apenas a satisfação do paciente com o atendimento recebido e não com todo o processo implicado nesse atendimento ${ }^{(21,24)}$. A falta de opção por outro serviço de saúde a ser utilizado também influencia na avaliação do nível de satisfação com o serviço recebido ${ }^{(25)}$. O direito à assistência à saúde, muitas vezes, não é percebido pelo paciente como tal, o que o faz sentir-se agradecido apenas por não ser maltratado ${ }^{(26)}$.

Os entrevistados estão mais insatisfeitos quanto aos indicadores de confiabilidade, com nítida insatisfação em relação ao cumprimento das atividades nos horários marcados (item 5) e também em relação à execução dos serviços no prazo prometido (item 8). Alguns estudos apontam esses dois itens como elementos centrais na percepção da satisfação dos pacientes de serviços públicos ${ }^{(10,22)}$. Existe um consenso de que um dos pontos débeis do serviço público é quanto ao tempo de espera prolongado ${ }^{(10)}$. Deve-se destacar que a confiabilidade juntamente com a segurança foram as consideradas pelos entrevistados como as mais importantes dimensões na avaliação da qualidade de um serviço de saúde.

Alguns autores afirmam que o nome "PACIENTES" está perfeitamente aplicado para designar as pessoas atendidas por serviços públicos de saúde ${ }^{(25)}$. De forma semelhante a esse trabalho, outros autores também encontram queixas freqüentes em relação ao cumprimento das atividades nos horá- 
rios marcados e em relação à execução dos serviços no prazo prometido, dentre outras ${ }^{(22,25)}$.

A segurança foi considerada a mais importante das dimensões da qualidade de serviço pela maioria dos entrevistados e, nesse sentido, o grau de insatisfação dos usuários foi muito baixo, sendo menor ainda com relação ao item 17: a equipe deve ter conhecimento adequado para responder às perguntas dos pacientes, fato este que difere dos achados de outros autores $^{(25)}$.

A satisfação dos pacientes com a atenção recebida é um dos indicadores da melhora da qualidade do serviço ${ }^{(10)}$. Alguns estudos confirmam que a administração hospitalar deve estar consciente da importância relativa de cada uma das 5 dimensões da qualidade de serviço na formação do nível de satisfação de seus pacientes ${ }^{(27)}$. Outro autor, em seu estudo, identificou o fornecimento de altos níveis de qualidade nos serviços como uma chave estratégica, inclusive aumentando a utilização de serviços das empresas de saúde em função da satisfação dos pacientes ${ }^{(28)}$.

\section{CONCLUSÕES}

Os dados obtidos neste estudo sugerem que, no geral, o grau de insatisfação com o serviço prestado pela instituição foi discreto, principalmente no aspecto segurança; porém permitiu detectar que é na confiabilidade que a assistência está mais deficitária, e, portanto, deve-se atuar, de forma prioritária, nesta dimensão, para melhorar a qualidade do serviço, através da implementação de ações, principalmente, no sentido de diminuir o tempo de espera, bem como no sentido de executar os serviços no prazo prometido. Posicionando-se os usuários do serviço público em posição central, a construção de novas lógicas nessa instituição deverá buscar formas mais humanizadas de compreender e atender às necessidades dos que a buscam.

Os dados obtidos neste estudo sugerem que monitorizar a qualidade do serviço, através do emprego periódico da escala SERVQUAL, permitiria não só planejar estratégias precisas de intervenção de alta efetividade nos serviços de saúde, como também monitorizar a resposta a essas ações.

Acreditamos que essas conclusões também se apliquem a outros serviços vinculados ao SUS e que, portanto, poderiam trazer benefícios para a melhora da qualidade da assistência médica no sistema como um todo.

\section{ABSTRACT}

Purpose: To identify the perception of the ophthalmic service quality provided for outpatients of the public healthcare system as well as to detect which actions should be considered necessary and priority in order to improve its quality. Methods: A quantitative descriptive study was carried out on 100 outpatients of the public healthcare system which were submitted to ophthalmic tests at Fundação Hilton Rocha - Belo Horizonte -
MG, from July $1^{\text {st }}-$ July $30^{\text {th }} 2004$. Individual interviews were carried out by giving the interviewees two structured questionnaires adapted from the modified SERVQUAL. This scale is in agreement with the reality of the studied institute. Results: The adapted SERVQUAL scale was submitted to statistical validation and it showed a suitable internal consistency index. In general terms, a slight general dissatisfaction was detected regarding ophthalmological service quality. The interviewees cared more about safety and reliability. A higher degree of dissatisfaction was detected mainly concerning fulfillment of procedures at scheduled appointments related to the execution of services within due timelimits. Conclusions: The institute is supposed to plan as well as carry out actions which lead to a general improvement in the patient's satisfaction regarding service quality and mainly reliability. Service quality monitoring through periodic use of the SERVQUAL scale will not only make it possible to plan highly precise and effective intervention strategies in these and in other healthcare services but it will also allow monitoring the responses to these actions. All these actions will contribute to the improvement of the service in the system as a whole.

Keywords: Marketing of health services; Ophthalmology; Quality of health care; Quality indicators, health care; Ambulatory care, Questionnaires/utilization

\section{REFERÊNCIAS}

1. Samara BS, Barros JC de. Pesquisa de marketing: conceitos e metodologia. 3a ed. São Paulo: Prentice Hall; 2002.

2. Marchetti RZ, Prado PHM. Um tour pelas medidas de satisfação do consumidor. RAE Rev Adm Empresas FGV. 2001;41(4):56-67.

3. Roncati VLC. Relação entre os atributos da qualidade de um serviço de reabilitação ambulatorial e os padrões do modelo de acreditação Joint Commission on accreditation of helth care organizations: a visão do cliente. [tese] São Paulo: Escola de Administração de Empresas de São Paulo; 2002.

4. Resende SP. Percepção dos clientes sobre a qualidade do serviço prestado por uma empresa no ramo de mineração e transportes: uma aplicação do instrumento SERVQUAL modificado [tese]. Belo Horizonte: Pontifícia Universidade Católica de Minas Gerais; 2004.

5. Lovelock C, Lauren W. Serviços: marketing e gestão. Tradução: Cid Knipel Moreira. São Paulo: Saraiva; 2001. p.113-20.

6. De Man S, Gemmel P, Vlerick P, Van Rijk P, Dierckx R. Patients' and personnel's perceptions of service quality and patient satisfaction in nuclear medicine. Eur J Nucl Med Mol Imaging. 2002;29(9):1109-17. Comment in: Eur J Nucl Med Mol. Imaging. 2003;30(3):472.

7. Geertzen JH, Gankema HG, Groothoff JW, Dijkstra PU. Consumer satisfaction in prosthetics and orthotics facilities. Prosthet Orthot Int. 2002;26(1):64-71.

8. Lim PC, Tang NK. A study of patients' expectations and satisfaction in Singapore hospitals. Int J Health Care Qual Assur Inc Leadersh Health Serv. 2000; 13(6-7):290-9.

9. Speller S, Ghobadian A. Change for the public sector I. Manag Serv Qual. 1993;3(5):29-34.

10. Prieto Rodríguez MA, March Cerda JC, López Fernandez LA. [The quality perceived by the users of health centers and by private insurance companies]. Aten Primaria. 1999;24(5):259-66. Spanish.

11. Brasil VS. Dimensões da qualidade percebida em serviços de saúde diferenciados. In: XVIII Encontro Anual da Associação Nacional de Programas de Pós-Graduação em Administração. Área Temática de Marketing; 1994. Anais. Curitiba, set 1991. v.7. p.143-66.

12. Parasuraman A, Berry LL, Zeithaml VA. Refinement and reassessment of the SERVQUAL scale. J Retailing. 1991;67(4):420-31. 
13. Parasuraman A, Berry LL, Zeithaml VA. SERVQUAL: A multiple-item scale for measuring consumer perceptions of service quality. J Retailing. 1988;64(1): $12-40$.

14. Malhotra NK. Pesquisa de Marketing. 3a ed. Porto Alegre: Bookman; 2001. $719 \mathrm{p}$.

15. Johnson R, Bhattacharyya G. Statistics principles and methods. New York: John Wiley \& Sons; 1986. 578p.

16. Johnson RA, Wichern DW. Applied multivariate statistical analysis, New Jersey: Prentice Hall; 1988. 607p.

17. Williams B, Coyle J, Healy D. The meaning of patient satisfaction an explanation of high reported level. Soc Sci Med. 1998;47(9):1351-9.

18. Bernhart M, Wiadnyana I, Wihardjo H, Pohan I. Patient satisfaction in developing countries. Soc Sci Med. 1999;48(8):989-96.

19. Velasco C, de la Quintana C, Jové G, Torres LA, Bailey P. [Quality of contraception services in El Alto, Bolivia]. Rev Panam Salud Pública. 1999; 5(6):411-22. Spanish.

20. Sinqh H, Haqq E, Mustapha N. Patient's perception and satisfaction with health care professionals at primary care facilities in Trinidad Tobago. Bull World Health Organ. 1999;77(4):356-60.
21. Ramirez- Sanchez TJ, Nájera-Aguillar P, Nigenda- Lopez G. [Perception of the quality of care in the health services in Mexico: perspective of the users]. Salud Pública Méx. 1998;40(1):3-12. Spanish.

22. Vicuña M. Nível de satisfacción y disfunciones percebidas sobre la calidad de atención de los servicios obstétricos. An Fac Med (Perú). 2002; 63(1):40-50.

23. Atkinson S, Ngwengwe A, Macwan'gi M, Ngulube T, Harpham T, O'Connell. The referral process and urban health care in sub-Saharan África: the case of Lusaka, Zâmbia. Soc Sci Méd. 1999;49(1):27-38.

24. Verheggen FW, Nieman FH, Reerink E, Kok GJ. Patient satisfaction with clinical trial participation. Int J Qual Health Care. 1998;10(4):319-30.

25. Franco SC, Campos GWS. [Quality-of-care assessment in a university hospital pediatric clinic]. Cad Saúde Pública. 1998;14(1):61-70. Portuguese.

26. Sanna MC. Avaliação da assistência ambulatorial de enfermagem segundo a percepção do cliente. Rev Esc Enfermagem USP 1993;27(1):133-49.

27. Cho WH, Lee H, Kim C, Lee S, Choi KS. The impact of visit frequency on the relationship between service quality and outpatient satisfaction: a South Korean study. Health Serv Res. 2004;39(1):13-33.

28. John J. Improving quality through patient provider information. J Health Care Mark. 1992;12(3):46-55.

\section{Congresso Norte-Nordeste de Oftalmologia}

\section{6 с 8 de Selembro de 2006}

\section{Multicenter SEBRAE São Luís - MA}

Av. dos Holandeses, $s / \mathrm{n}^{\circ}$ - Térreo do Hotel Belagio CEP 65075-650 - São Luís - MA Telefax: (98) 3213-6350 / (98) 8126-5958 com Maria Luiza E-mail: marialuiza@taguatur.com.br Home-page: offalmo2006nne.com.br 